\title{
Silane-modified surfaces in specific antibody-mediated cell recognition
}

\author{
Karolina Sterzynska $a^{1,2^{*}}$, Joanna Budna ${ }^{1^{*}}$, Emilia Frydrych-Tomczak ${ }^{1,3}$, \\ Grzegorz Hreczycho $^{4}$, Agnieszka Malinska ${ }^{1,2}$, Hieronim Maciejewski ${ }^{3,4}$, Maciej Zabel ${ }^{1,2}$
}

\author{
${ }^{1}$ Wroclaw Research Center EIT+, BioMed, Wroclaw, Poland \\ ${ }^{2}$ Department of Histology and Embryology, Poznan University of Medical Sciences, Poznan, Poland \\ ${ }^{3}$ Poznan Science and Technology Park, Adam Mickiewicz University Foundation, Poznan, Poland \\ ${ }^{4}$ Faculty of Chemistry, Adam Mickiewicz University, Poznan, Poland \\ *These authors contributed equally
}

\begin{abstract}
The immobilization of antibodies on various surfaces has been the subject of advanced research in various immunoassay-based diagnostic devices. The physical and chemical stabilities of the immobilized antibodies on a solid surface still cause many problems because upon immobilizing antibody molecules, the antigen-binding ability usually decreases. The silanization of surfaces with organosilanes carrying chemically active groups such as (3-aminopropyl)triethoxysilane (APTES) can accommodate these antigen-binding molecules in an appropriate orientation so that their functionality and binding activity are essentially retained. In this study, n-butyltrimethoxysilane (BMS) and 3-(octafluoropentyloxy)-propyltriethoxysilane (OFPOS) were used as "blocking silanes". The aims of this study were to compare the effectiveness of specific antibody binding of APTES, APTES + BMS and APTES + OFPOS and to characterize the modified surfaces by contact angle measurements and immunofluorescence measurements prior to and after immobilizing proteins. Additionally, we have evaluated the functionality of the immobilized antibodies by their abilities to bind EpCAM-positive human colon adenocarcinoma cell line (LoVo) and EpCAM-negative mouse embryonic fibroblast cell line (3T3). Cell enumeration was conducted on the basis of DAPI-positive signals and recorded using a confocal laser scanning biological microscope. The results of our study showed that the immobilization capability and reactivity of APTES, APTES + BMS and APTES + OFPOS differ. The modification of APTES with unreactive silanes (BMS, OFPOS) is recommended to improve the antibody binding efficiency. However, using OFPOS resulted in more effective antibody and cell binding, and it appears to be the most useful compound in specific antibody-mediated cell recognition. (Folia Histochemica et Cytobiologica 2014, Vol. 52, No. 3, 250-255)
\end{abstract}

Key words: silanes; APTES; antibody immobilization; cell binding; cell culture; EpCAM; immunofluorescence; contact angle analysis

\section{Introduction}

In recent years, the immobilization of antibodies on various surfaces has been the subject of advanced research in immunoassay-based diagnostic devices used

\footnotetext{
Correspondence address: K. Sterzynska, Ph.D. Department of Histology and Embryology Poznan University of Medical Sciences Swiecickiego 6 St., 60-781 Poznan tel.: +4861 85464 55; fax: +48618546440 e-mail: k.olejniczak@ump.edu.pl
}

for detecting rare-occurring cells, including circulating and disseminating tumor cells (CTCs, DTCs) [1-3]. The physical and chemical stabilities of the immobilized antibodies still cause many problems because upon immobilization, their binding activity decreases as the result of their random orientation (e.g. the blocking of Fab ends) and the steric hindrance of the antibody [4]. Many silicon-based technologies have been developed and applied as sensitive and selective biosensors and biomedical devices, e.g. nanowires or nanoparticles [5-7]. Among these technologies, silanization with organosilanes carrying chemically active 


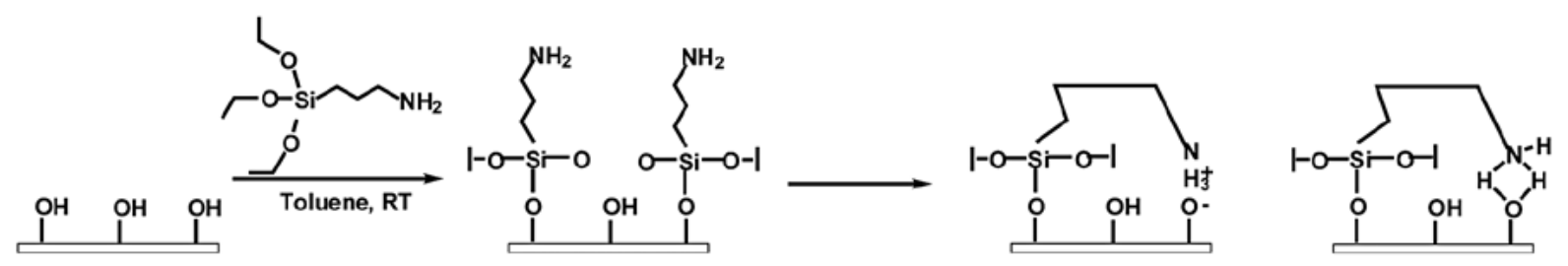

Figure 1. Structures of silanes used in this study. A. 3-aminopropyltriethoxysilane (APTES); B. APTES with n-butyltrimethoxysilane (BMS); C. APTES with 3-(octafluoropentyloxy)propyltriethoxysilane (OFPOS)

groups $[8,9]$, such as (3-aminopropyl)triethoxysilane (APTES) (Figure 1A), is one of the most common and simple methods. Antibodies are immobilized on modified silica surfaces by forming covalent bonds between protein functional groups and the complementary coupling groups of organosilane [10, 11]. APTES can accommodate these molecules in an appropriate orientation so that their functionality and binding activity are essentially retained $[8,12]$. However, the high reactivity of amino groups and the presence of unbound silanol groups on the surface enable the interaction of these groups and the formation of hydrogen bonds. Those effects result in restricted access to free amino groups and the reduced immobilization of biomolecules on the surface. One of the ways to counteract such a situation is to block free silanol groups by an additional silanization with an unreactive silane, e.g. alkylsilane. Such a procedure results in the rupture of hydrogen bonds and the "straightening" of aminopropyl groups, thus increasing the access to amino groups on the modified surface $[13,14]$. In this study, n-butyltrimethoxysilane (BMS) and 3-(octafluoropentyloxy)-propyltriethoxysilane (OFPOS) were used as "blocking silanes" to prevent amino groups bending towards unreacted silanols (Figure 1B, C). Because the effectiveness of specific antibody binding is of considerable interest, the aims of this study were to compare APTES, APTES + BMS and APTES + OFPOS as the silylating agents and to characterize the modified surfaces with those organosilanes prior to and after immobilizing proteins. Additionally, we have evaluated the functionality of the immobilized antibodies by measuring their abilities to bind specific surface antigens of cultured cancer cells.

\section{Material and methods}

Preparation of silanized glass surfaces. Glass slides were silanized using three organofunctional trialkoxysilanes: 3-aminopropyltriethoxysilane - APTES (> 99\%, Sigma-Aldrich, St. Louis, MO, USA) and APTES with the addition of n-butyltrimethoxysilane - APTES + BMS (BMS 97\%, ABCR, Karlsruhe, Germany) and 3-(octafluoropentyloxy) propyltriethoxysilane - APTES + OFPOS (OFPOS, Poznan Incubator of Chemical Technologies, Poznan, Poland). The glass slides $(26 \times 76 \mathrm{~mm}$, Knittel Glass, Braunschweig, Germany) were first cleaned by incubating in 1:1 concentrated $\mathrm{HCl}: \mathrm{MeOH}$ for $30 \mathrm{~min}$, rinsing with distilled water, sonicating in acetone for $5 \mathrm{~min}$ and drying in a stream of $99.999 \%$ nitrogen (Linde Gaz, Bielsko Biala, Poland). The cleaned glass slides were sonicated with ultrasound frequency $37 \mathrm{kHz}$ (Elmasonic S60H, Singen Germany) in a $2 \%$ solution of APTES in toluene for $1 \mathrm{~h}$. Then, the slides were rinsed in toluene, methanol-toluene (1:1) and methanol in an ultrasonic bath, in each case for $5 \mathrm{~min}$. The slides were dried in a microcentrifuge and placed in an oven at $110^{\circ} \mathrm{C}$ for $1 \mathrm{~h}$. The glass slides modified with APTES in the presence of BMS or OFPOS were sonicated in a $2 \%$ toluene solution for $1 \mathrm{~h}$ directly after the amino-functional silane modification. Then, the slides were cleaned in an ultrasonic bath with toluene, toluene-methanol and methanol; dried in a microcentrifuge; and baked at $110^{\circ} \mathrm{C}$ for $1 \mathrm{~h}$.

Contact angle measurement. Static contact angle $(\theta)$ measurements were performed using the DSA $100 \mathrm{E}$ auto goniometer from Krüss (Hamburg, Germany). The measurements were made on sessile drops of water $(3 \mathrm{~mL}$ droplets) by measuring the tangent angle to the drop at its intersection with the slide surface. Advancing contact angles were determined by performing a minimum of eight independent measurements and are presented as means \pm standard deviation.

Antibody binding. Monoclonal anti-EpCAM (epithelial cell adhesion molecule) antibody was chosen as a specific protein because of its selective binding affinity toward the corresponding antigen on the surface of cancer cells of epithelial origin. APTES-covered glass surfaces were coated with $25 \mu \mathrm{g} / \mathrm{mL}$ and $5 \mu \mathrm{g} / \mathrm{mL}$ mouse monoclonal anti-EpCAM antibody (EBA-1, Santa Cruz Biotechnology, Dallas, TX, USA) in $0.05 \mathrm{M}$ phosphate-buffered saline (PBS) at $\mathrm{pH}$ 7.4, or with PBS alone (for the control area), for $1 \mathrm{~h}$ at room temperature (RT). The incubation was followed by three washes with PBS. Next, the free protein-binding sites of the glass slides were blocked by incubating with a $3 \%$ bovine serum albumin (BSA) solution in PBS for $1 \mathrm{~h}$ at 
Table 1. Mean values of contact angles for glass slides covered with silanes

\begin{tabular}{|l|c|c|c|}
\hline Silane/surface & $\theta\left[^{\circ}\right]$ without antibody & $\theta\left[^{\circ} \mathbf{~} \mathbf{5} \boldsymbol{\mu} \mathbf{g} / \mathbf{m L}\right.$ anti-EpCAM antibody & $\theta\left[^{\circ} \mathbf{~} \mathbf{2 5} \boldsymbol{\mu \mathbf { g }} / \mathbf{m L}\right.$ anti-EpCAM antibody \\
\hline APTES & $73.3 \pm 0.96$ & $64.2 \pm 1.88$ & $59.2 \pm 0.65$ \\
\hline APTES + BMS & $77.5 \pm 1.12^{*}$ & $61.9 \pm 3.44$ & $59.8 \pm 1.33$ \\
\hline APTES + OFPOS & $101.6 \pm 2.44^{* * \#}$ & $66.8 \pm 1.83^{\&}$ & $53.3 \pm 6.12^{\&}$ \\
\hline
\end{tabular}

Data present mean values \pm standard deviation based on three independent cell culture experiments and three measurements for each type of glass slides silanized with APTES (3-aminopropyltriethoxysilane), APTES + BMS (n-butyltrimethoxysilane) and APTES + OFPOS (3-(octafluoropentyloxy) propyltriethoxysilane) before and after antibody immobilization. The contact angle of purified control glass slides was about $7^{\circ}$. ${ }^{*}$ Significantly different from APTES (no antibodies), $\mathrm{p}<0.01$; " significantly different from APTES + BMS (no antibodies), $\mathrm{p}<0.01$; ${ }^{\&}$ significantly different from APTES + OFPOS (no antibodies), $\mathrm{p}<0.01$

RT. Blocking was followed by three washes with PBS. The EpCAM protein was detected with a $2 \mu \mathrm{g} / \mathrm{mL}$ FITC-labeled secondary anti-mouse antibody (MFP488, goat anti-mouse $\mathrm{IgG}$, MoBiTec, Goettingen, Germany) solution in PBS for $1 \mathrm{~h}$ at RT, followed by three washes with PBS. The effectiveness of the antibody binding was analyzed by a water contact angle measurement (i.e. goniometer analysis) and measurements of fluorescence intensity using an Olympus Fluoview i10 confocal laser scanning biological microscope. Images were captured from randomly selected regions of interest (ROIs) and recorded by an analog integration detection system. The data from the recorded images were converted into histograms and presented as average values. The fluorescence intensity was measured from 5 areas of $1.5 \mathrm{~mm}^{2}$ each, and the estimations were performed three times for each silane.

Evaluation of antibody binding. The effectiveness of the anti-EpCAM antibody binding to the APTES surfaces was evaluated by the ability of the antibody to bind specific cultured cells. Two cell lines were used in this study: an EpCAM-positive human colon adenocarcinoma cell line (LoVo, ATCC, Manassas, VA, USA) and an EpCAM-negative mouse embryonic fibroblast cell line (3T3, ATCC, Manassas, VA, USA). Cells were routinely cultured in DMEM medium supplemented with $5 \%$ sterile-filtered fetal bovine serum (FBS), L-glutamine and 1\% antibiotic-antimycotic solution (all from Sigma-Aldrich). Cell lines were grown to near confluence, harvested and counted. In this experiment, 100,000 cells per mL were used. APTES glass slides, covered with anti-EpCAM antibodies in concentrations of $25 \mu \mathrm{g} / \mathrm{mL}$ or $5 \mu \mathrm{g} / \mathrm{mL}$, or without antibodies, were incubated with cells. The experiment was conducted with a Mini Rocker MR-1 Shaker (Biosan, Riga, Latvia), which provided a regulated rocking motion and mixed the cell suspension for $30 \mathrm{~min}$ at RT. Afterwards, slides were washed three times with PBS and sealed with DAPI (4',6-diamidino-2-phenylindole) mounting medium (Ultra Cruz DAPI Mounting Medium, Santa Cruz Biotechnology, Dallas, TX, USA). Cell enumeration was conducted on the basis of DAPI-positive signals and recorded using a confocal laser scanning biological microscope (Olympus Fluoview i10, Olympus, Tokyo, Japan) in an area of $48.6 \mathrm{~mm}^{2}$. Each experiment was performed three times. The number of cells counted in each control area was taken as a value of 1 , and the counts of the tested areas were presented as relative fold changes (either increases or decreases) compared with the respective control.

Statistical analysis. Statistical analysis was performed using one-way ANOVA with a post-hoc test of least significant difference.

\section{Results}

The efficiency of antibody binding was evaluated by the contact angle and fluorescence intensity measurements. The contact angles were significantly higher after silanization with APTES, APTES + BMS and APTES + OFPOS, which showed unambiguously that the glass slide surface was modified. However, the contact angles differed depending on the surface properties of the employed silane. Further surface modification of the glass slides using BMS and OFPOS resulted in an increase in the contact angle ( $p<0.01$, Table 1). Similar analysis was performed for silanized glass slides after the incubation with $25 \mu \mathrm{g} / \mathrm{mL}$ and $5 \mu \mathrm{g} / \mathrm{mL}$ of anti-EpCAM antibodies (Table 1). In each case, a decrease in the contact angle was observed, suggesting that the antibodies were successfully immobilized. However, the most significant difference in contact angle values, measured prior to and after antibody immobilization, was observed for the APTES + OFPOS slide ( $p<0.01$, Table 1$)$.

Immunofluorescence analysis, used as a second method of evaluating the antibody binding, also showed differences between the examined compounds. The amount of immobilized antibody varied significantly depending on its applied concentration for the APTES + OFPOS slide ( $p<0.001$, Figure 2). This result suggests that anti-EpCAM antibodies bound more effectively to APTES + OFPOS modified glass 


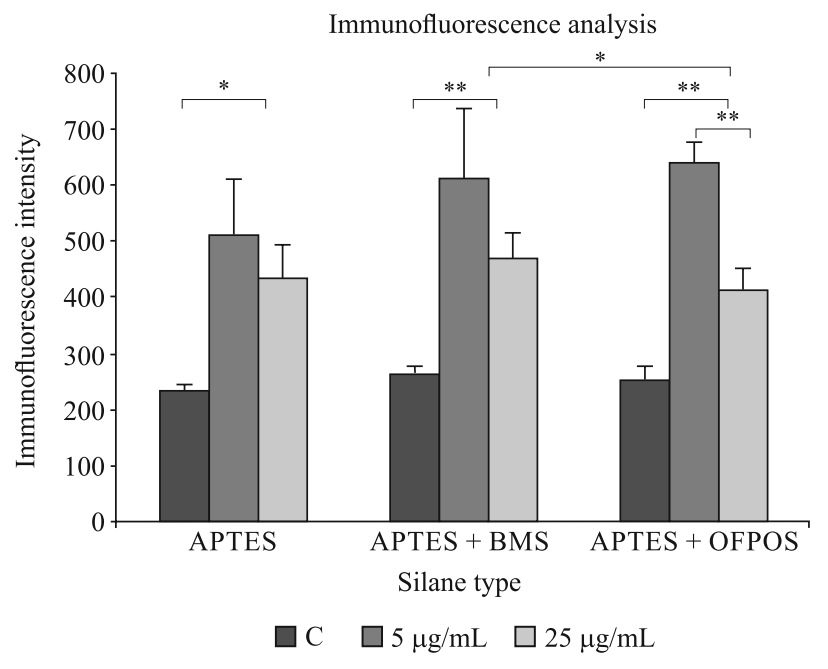

Figure 2. Immunofluorescence intensity of silanized glass slides for areas covered with Ep-CAM antibodies $(5 \mu \mathrm{g} / \mathrm{mL}$ and $25 \mu \mathrm{g} / \mathrm{mL}$ ) and for control area without antibody (C). $\mathrm{Y}$ axis: immunofluorescence intensity in arbitrary units (AU). Histograms present mean values and bars standard deviation based on three independent cell culture experiments and three measurements for each type of glass slides. $* \mathrm{p}<0.05, * * \mathrm{p}<0.001$

surfaces than to other examined silanes. Moreover, we found a difference between APTES + BMS and APTES + OFPOS, indicating that APTES + OFPOS was more effective in binding the EpCAM antibodies than the commonly used APTES + BMS $(p<0.05$, Figure 2). The combination of these two silanes (APTES + OFPOS) on the glass slides resulted in a better binding of antibodies than in the case of APTES alone and in combination with BMS. This may be explained by the presence of strongly electronegative fluorine in APTES + OFPOS silane, manifesting a very low polarizability and having a minimal ability to interact with the antibody surface $[15,16]$.

Notably, an increase in antibody concentration from $5 \mu \mathrm{g} / \mathrm{mL}$ to $25 \mu \mathrm{g} / \mathrm{mL}$ did not result in a higher fluorescence intensity (Figure 2) for that silane. This result agreed with the phenomenon of the antibody-binding capacity. An increase in the antibody concentration affects the packing density and leads to the steric hindrance of antigen binding $[17,18]$.

\section{Discussion}

The immobilization of antibodies on various surfaces has become a subject of great interest to many researchers. In addition to the physical and chemical aspects of the antibodies' attachment, their diagnostic utility is also considered, e.g. for detecting rarely occurring cells [1-3]. In our experiment, the EBA-1 antibody was

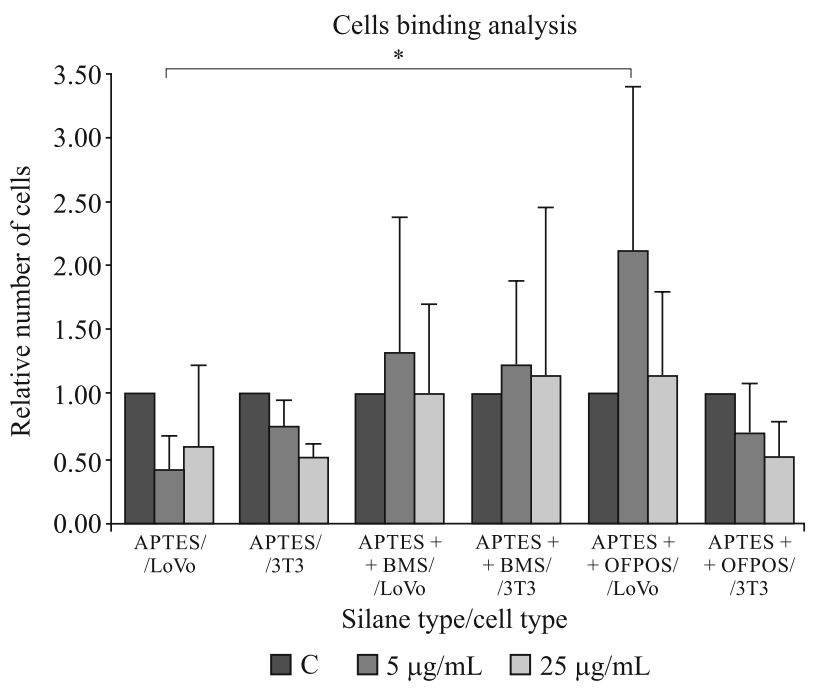

Figure 3. Cell binding ability of silanized glass slides for areas covered with antibodies $(5 \mu \mathrm{g} / \mathrm{mL}$ and $25 \mu \mathrm{g} / \mathrm{mL})$ and for control area (without antibody, C). Number of cells from tested areas is presented as relative fold change in comparison to the respective control (assumed to have value of 1). Other details as in the description of Figure 2. ${ }^{*} \mathrm{p}<0.05$

chosen to be immobilized on the silanized surfaces, as it showed the most specific and strong signal for the EpCAM-positive cell lines [19]. Therefore, two different types of cells, LoVo (human epithelial cells), which are EpCAM-positive, and 3T3 (mouse mesenchymal cells), which are EpCAM-negative, were used to study the binding capacity of the antibodies. The highest number of attached EpCAM-positive LoVo cells was observed for the APTES+OFPOS glass slides covered with a $5 \mu \mathrm{g} / \mathrm{mL}$ anti-EpCAM antibody area. The result was significant for APTES and APTES + OFPOS $(p<0.05$, Figure 3, Figure 4 B, H). The higher concentration of antibody $(25 \mu \mathrm{g} / \mathrm{mL})$ did not result in a higher number of attached cells (Figure 3, Figure 4G-I). This result can be explained by the steric requirement of the biomolecules since a high concentration of antibodies was found to decrease their ability to bind cells $[12,18]$.

None of the two applied concentrations of antibody affected the number of LoVo and 3T3 cells attached to APTES and APTES + BMS (Figure 3, Figure 4A-F). As expected, the binding capability of EpCAM-negative 3T3 cells was insignificant for all of the tested silanes, independent of the antibody concentration (Figure 3). Even if both types of 'blocking silanes' (BMS, OFPOS) could prevent the bending of amino groups and facilitate proper antibody binding [14], only the APTES + OFPOS combination appeared more useful for cell recognition and binding. A remarkably higher number of attached cells was observed (Figure $4 \mathrm{~A}-\mathrm{I}$ ), which indicated that the 

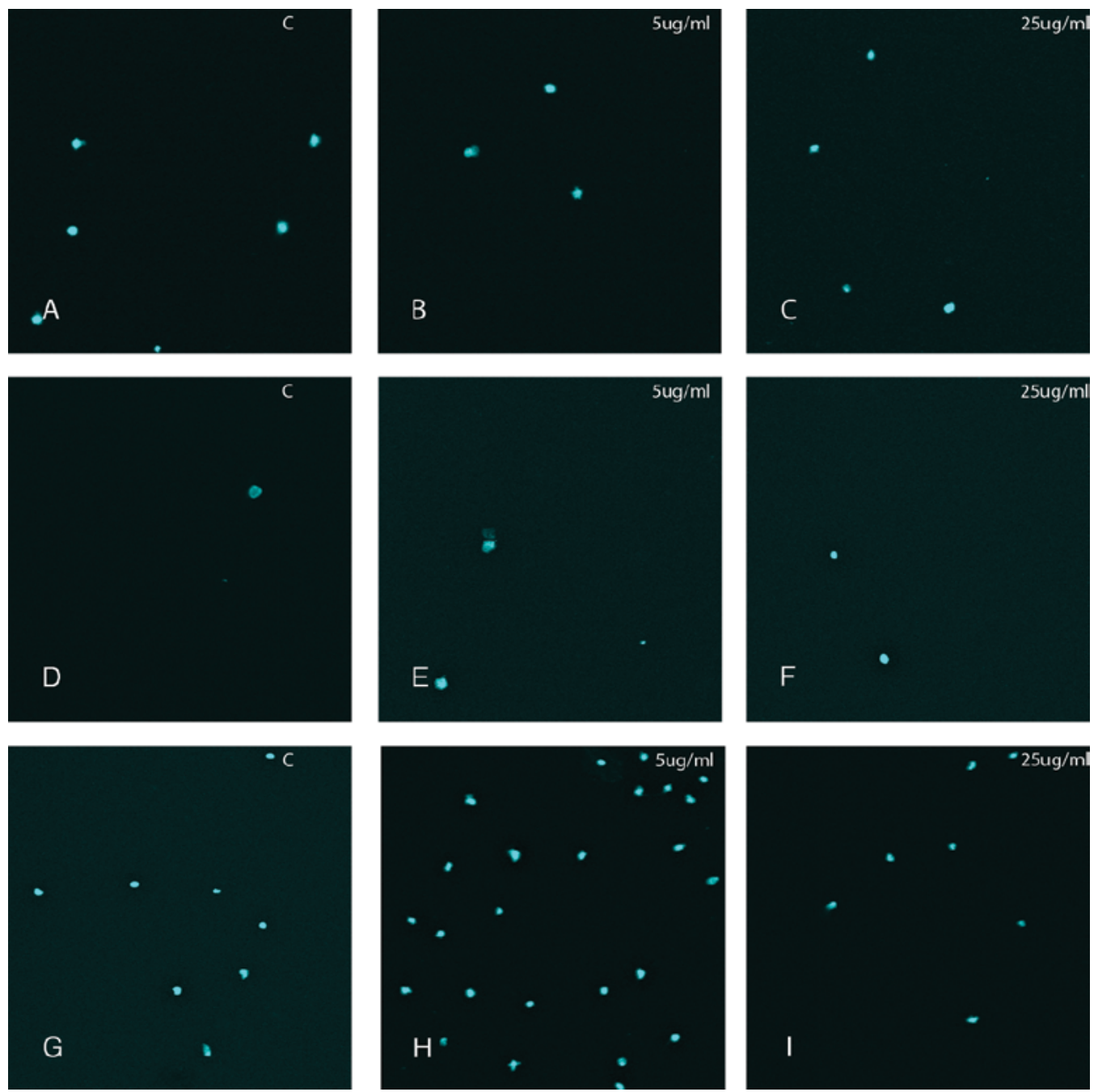

Figure 4. LoVo cell binding ability of silanized glass slides for areas covered with Ep-CAM antibodies applied at indicated concentrations or without antibodies. C - control area (without antibody). Upper row: A, B, C - APTES; middle row: D, E, F - APTES + BMS; lower row: G, H, I - APTES + OFPOS. Immunofluorescence technique: nuclei stained with DAPI (one signal corresponds to one cell). Objective magnification $\times 10$

antibodies immobilized on the APTES + OFPOS surface were more available for binding to cell surface antigens.

To summarize, the contact angle measurements and the fluorescence microscopy and cell-binding ability tests showed that the immobilization capability and reactivity of APTES, APTES + BMS and APTES + OFPOS differ. The modification of APTES-covered surfaces with unreactive silanes (BMS, OFPOS) is recommended to improve the antibody-binding efficiency.

\section{Acknowledgments}

The research was supported by the Wroclaw Research Centre EIT+ under the project "Biotechnologies and advanced medical technologies" - BioMed (POIG.01.01.02-02-003/08) financed from the European Regional Development Fund (Operational Programme Innovative Economy, 1.1.2).

\section{References}

1. Iijima M, Matsuzaki T, Yoshimoto N, Niimi T, Tanizawa K, Kuroda S. Fluorophore-labeled nanocapsules displaying IgG Fc-binding domains for the simultaneous detection of multiple antigens. Biomaterials. 2011;32:9011-9020.

2. Pantel K, Brakenhoff RH, Brandt B. Detection, clinical relevance and specific biological properties of disseminating tumor cells. Nat Rev Cancer. 2008;8:329-340.

3. Budna J, Sterzynska K, Frydrych-Tomczak E, Maciejewski $\mathrm{H}$, Zabel M. Linkers for rare cancer cells isolation. Virchows Arch. 2013;463:325.

4. Halliwell CM. Nanoanalytical measurement of protein orientation on conductive sensor surfaces. Analyst. 2004;129:1166-1170. 
5. Cui Y, Wei Q, Park H, Lieber CM. Nanowire nanosensors for highly sensitive and selective detection of biological and chemical species. Science. 2001;293:1289-1292.

6. Tan W, Wang K, He X et al. Bionanotechnology based on silica nanoparticles. Med Res Rev. 2004;24:621-638.

7. Zheng G, Patolsky F, Cui Y, Wang WU, Lieber CM. Multiplexed electrical detection of cancer markers with nanowire sensor arrays. Nat Biotechnol. 2005;23:1294-1301.

8. Uszczyńska B, Ratajczak T, Frydrych E et al. The application of click chemistry to the production of DNA microarrays. $L a b$ Chip 2012;12:1151-1156.

9. De Vos K, Bartolozzi I, Schacht E, Bienstman P, Beats R. Silicon-on-Insulator microring resonator for sensitive and label-free biosensing. Opt Express. 2007;15:7610-7615.

10. MacBeath G, Schreiber SL. Printing proteins as microarrays for high-throughput function determination. Science. 2000;289:1760-1763.

11. Wang ZH, Jin G. Silicon surface modification with a mixed silanes layer to immobilize proteins for biosensor with imaging ellipsometry. Colloid Surf B. 2004;34:173-177.

12. Couston RG, Lamprou DA, Uddin S, Walle CF. Interaction and destabilization of a monoclonal antibody and albumin to surfaces of varying functionality and hydrophobicity. Int J Pharm. 2012;438:71-80.

13. Kim J, Seidler P, Wan L, Fill C. Formation, structure and reactivity of amino-terminated organic films on silicon substrates. J Colloid Int Sci. 2009;329:114-119.
14. Kamisetty NK, Pack SP, Nonogawa M, Devarayapalli KC, Kodaki T, Makino K. Development of an efficient amine-functionalized glass platform by additional silanization treatment with alkylsilane. Anal Bioanal Chem. 2006;386: 1649-1655.

15. Kamisetty NK, Pack SP, Nonogawa M, Devarayapalli KC, Kodaki T, Makino K. Additional alkylsilanization of aminosilane-modified glass slide: effect of alkylsilane structure for enhancing surface amine functionality. Chem Lett. 2007;36:322-323.

16. Biffinger JC, Kim HW, DiMagno SG. The polar hydro-phobicity of fluorinated compounds. Chembiochem. 2004;5:622-627.

17. Xu H, Zhao XB, Grant G, Lu JR, Williams DE, Penfold J. Orientation of a monoclonal antibody adsorbed at the solid/ /solution interface: a combined study using atomic force microscopy and neutron reflectivity. Langmuir. 2006;22: 6313-6320.

18. Xu H, Zhao XB, Lu JR, Williams DE. Relationship between the structural conformation of monoclonal antibody layers and antigen binding capacity. Biomacromolecules. 2007;8:2422-2428.

19. Sterzynska K, Kempisty B, Zawierucha P, Zabel M. Analysis of the specificity and selectivity of anti-EpCAM antibodies in breast cancer cell lines. Folia Histochem Cytobiol 2012;50:534-541. 